\title{
Programmable optical wave form shaper on a microchip
}

\author{
Kabir Udeshi, Kai-Hsiu Liao, Long Que, a) \\ Yogesh B. Gianchandani, and Almantas Galvanauskas ${ }^{\text {b) }}$ \\ College of Engineering, University of Michigan, Ann Arbor, Michigan 48109-2122
}

(Received 20 November 2005; accepted 11 June 2006; published online 21 July 2006)

\begin{abstract}
This letter reports a device for in-fiber programmable optical pulse shaping, that consists of a chirped fiber grating and a micromachined array of Si actuators integrated on a $1 \times 5 \mathrm{~mm}^{2}$ chip. The pulse spectrum is longitudinally imaged inside a chirped fiber Bragg grating, thus permitting spectral components to be accessed inside the fiber by individual actuators. The demonstration of controlled optical pulse spectrum and temporal-width changes shows that the silicon microactuators, fabricated using a standard lithographic process, can tune the local refractive index of the grating by inducing localized fiber core strain gradients at a rate of $>1 \mu$ Strain per $1 \mathrm{~mW}$ of actuator driving power. () 2006 American Institute of Physics. [DOI: 10.1063/1.2222247]
\end{abstract}

The programmable shaping of optical wave forms is needed for a number of scientific studies - the most notable of which are the coherent control of chemical reactions ${ }^{1}$ and quantum computing ${ }^{2}$ - and has many potential applications ${ }^{3}$ such as optical signal processing, optical communications, radar arrays, and ultrashort pulse lasers. Traditional pulse shapers use a diffraction grating (that provides spatial dispersion) and a combination of lenses to spatially image the pulse spectrum in a Fourier plane of the device. The Fouriertransformed light in this plane is passed through a spatial light modulator (SLM), such as a mask, a liquid crystal modulator, an acousto-optic modulator, or a deformable or a micromachined mirror, allowing the programmable modification of pulse spectral amplitude and phase and, consequently, the temporal shape of a recombined wave form. ${ }^{3}$ The main practical drawback of this approach is associated with its reliance on spatial dispersion effects: such devices require complex tolerance-sensitive optical alignment and, therefore, are quite challenging from an engineering and manufacturing perspective. For example, the micromirror based dispersion compensator reported in Ref. 4 illustrates the inherent spatial complexity of this approach. Here we demonstrate a micromachined platform for fiber-optic pulse shaping in which light is controlled inside an optical fiber without resorting to external spatial beam manipulation, thus permitting compact and robust programmable light-control technology.

The device uses an on-chip microactuator array, which produces a multitude of independently controllable local strain gradients in an embedded chirped fiber Bragg grating (CFBG), which locally affect the refractive index. In a chirped fiber Bragg grating, reflected-light wavelength satisfies the local Bragg condition: $\lambda_{B}(z)=2 n(z) \Lambda(z)$, where $\lambda_{B}(z)$ is the wavelength reflected at position $z, \Lambda(z)$ is the local grating period, and $n(z)$ is the local effective refractive index for the propagating mode in the fiber core, ${ }^{5}$ i.e., in a strongly chirped grating the reflection of each spectral component becomes localized at different positions along the grating. Consequently, the control of local $n(z)$ with a microactuator array enables the programmable control of wavelength-dependent

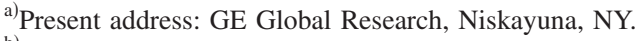

${ }^{b)}$ Electronic mail: almantas@umich.edu
}

phase shifts, thus permitting adjustable dispersion control, variable time delays, and programmable optical wave form shaping. We show here that, by using the electrothermal microactuators, ${ }^{6}$ a localized and controlled amount of force may be applied to a CFBG. It is known that strain can modify fiber core refractive index ${ }^{7}$ and shift the fiber grating Bragg wavelength reflected in this local region at a rate of $\sim 1.2 \mathrm{pm} / \mu$ Strain (Ref. 8) or, in terms of the corresponding refractive index change, $\sim 1.2 \times 10^{-6} / \mu$ Strain. Through the current applied to each individual electrothermal actuator in the array, the magnitude of the force produced by the actuator and, consequently, the local Bragg wavelength shift can be controlled, thus allowing multiple spectral components along the length of the CFBG to be addressed.

One possible pulse shaper implementation is to use a pair of linearly chirped FBGs oriented with opposite spatial chirp directions and connected to all-fiber circuitry through optical circulators (Fig. 1). The first grating stretches the incident bandwidth-limited pulse, and the second one compresses the stretched pulse back to the bandwidth-limited duration. Maintaining reciprocity between pulse stretching and compressing requires (i) both chirped gratings to be identical to each other and (ii) the grating chirp to be sufficiently large. The pulse shaping of the recompressed pulse then can be achieved if this reciprocity is "broken" by modulating the refractive index profile in one of the gratings.

In our setup, it is the pulse-compressing CFGB that is located between arrays of electrothermal microactuators ${ }^{6}$ [Fig. 2(b)]. The microactuators are constructed from suspended V-shaped beams, clamped at their two ends to anchors. When a potential is applied across the ends of these beams, the current through them causes Joule heating and consequent expansion. The apex of the expanded beam is pushed outward, generating a displacement or force. These microactuators generate rectilinear displacements with forces up to the millinewton range and can be fabricated from any material that is electrically conductive and has sufficient mechanical strength. Typical electrothermal microactuators have operating frequencies from dc to the kilohertz range. The microactuator array shown in Fig. 2(b) has overall dimensions of $5 \times 1 \mathrm{~mm}^{2}$. It consists of a total of 75 actuators spaced at $60 \mu \mathrm{m}$ intervals and is fabricated from doped silicon using deep reactive ion etching (DRIE). The two-mask process results in $50 \mu \mathrm{m}$ thick devices bonded to a glass 


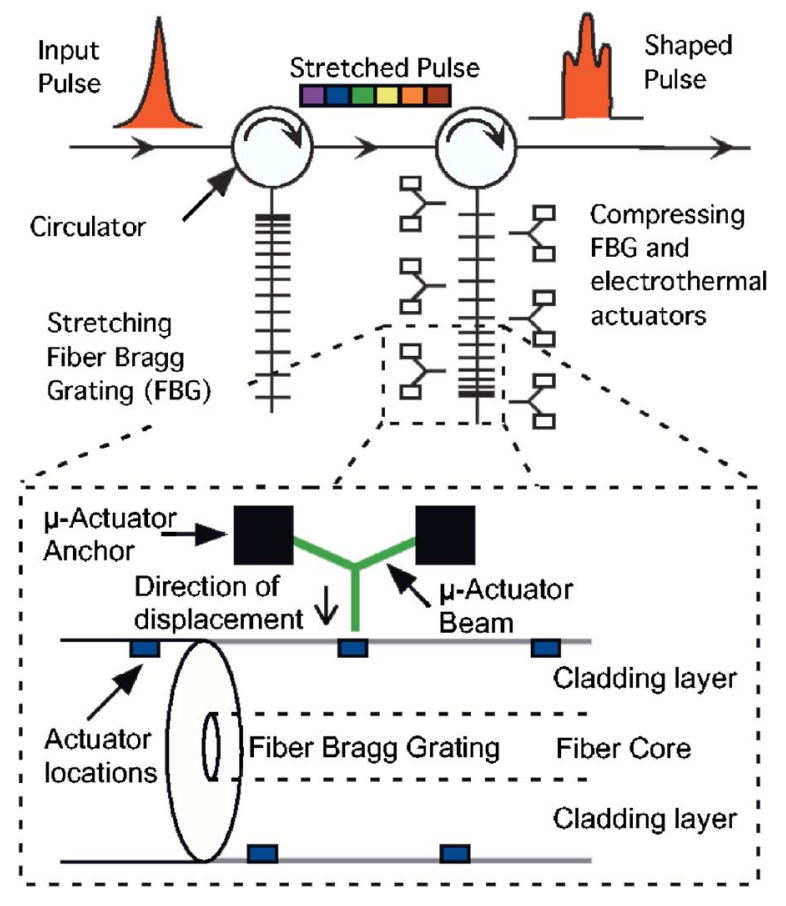

FIG. 1. Schematic of two-grating arrangement for programmable optical pulse shaping using a pair of reciprocally oriented identical chirped fiber gratings. An array of electrothermal microactuators locally alter the refractive index of a fiber Bragg grating, hence addressing individual components of the optical spectrum along the length of the grating.

substrate. A CFBG is inserted into the groove between the actuator arrays and then clamped in place with an adhesive. The dimensions of the groove are chosen so that the $80 \mu \mathrm{m}$ outer-diameter fiber fits snugly in the gap, with $5 \mu \mathrm{m}$ wide actuator tips touching the fiber.

Linearly chirped fiber Bragg gratings with $\sim 5 \mathrm{~nm}$ spectral bandwidths at $1550 \mathrm{~nm}$ central wavelength and $4.5 \mathrm{~mm}$
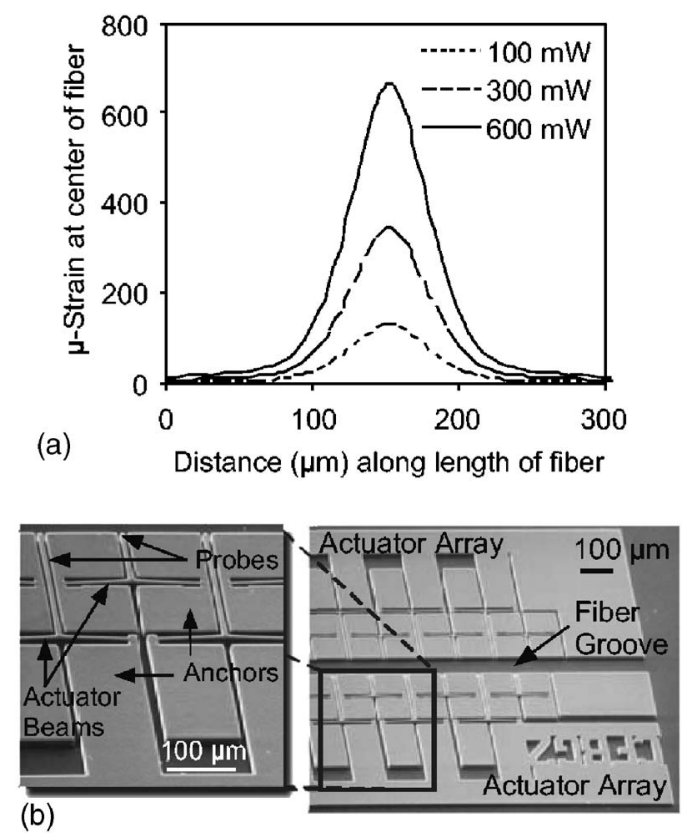

FIG. 2. (a) Simulated values of strain generated in the fiber using a single microactuator at various electric-power power levels. In this example $5 \mu \mathrm{m}$ wide actuator is positioned at $z=150 \mu \mathrm{m}$. (b) A scanning electron microscope (SEM) image of the actuator array, consisting of 75 actuators, along with the fiber groove into which the CFBG is inserted. Each actuator can be individually controlled and addressed.

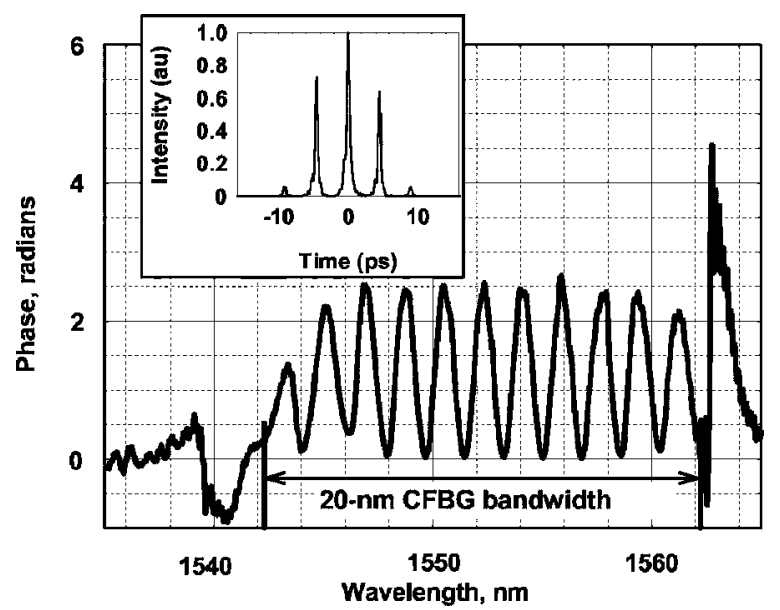

FIG. 3. Numerically simulated shaped-pulse spectral phase and temporal profile (inset) for a $20 \mathrm{~nm}$ bandwidth and $4.5 \mathrm{~mm}$ long CFBG when a sinusoidal modulating current pattern is applied along the 75-element MEMS array: $P(N)=150[\mathrm{~mW}] \sin (2 \pi N / 7)+150[\mathrm{~mW}]$, where $N$ is the actuator number along the array.

length were used. The gratings were apodized, providing a relatively smooth reflection spectrum profile. Grating reflectivity was $\sim 50 \%$. The laser pulses were generated using an Er-doped mode-locked fiber laser. The actuators in the array were individually addressed from 1 to 75 along the length of the grating.

The reflection-spectrum amplitude and phase of this chirped fiber grating embedded into this micro-electromechanical system (MEMS) array has been modeled using the effective-index method, ${ }^{9}$ with the actual apodization profile of the fabricated gratings included in the model. The calculation of the fiber grating response to the action of a single or multiple MEMS actuators included both mechanical and optical effects. The spatial strain profile induced inside the fiber by each microactuator is calculated using a finite element analysis. Examples of strain profiles along the fiber core induced by an individual actuator driven at different electric powers are shown in Fig. 2(a), predicting that with the present actuator design, $\sim 1 \mu \mathrm{Strain} / \mathrm{mW}$ is achievable at the "peak" of the bell-shaped $80 \mu \mathrm{m}$ full width at half maximum (FWHM) in-core strain distribution. We estimate that up to an order of magnitude reduction in driving power could be achieved in the future with optimized MEMSactuator structures. The in-core refractive-index change distribution is calculated using known elasto-optic coefficients for fused silica glass and then included into the effectiveindex model of the chirped grating to calculate the exact effect of each individual actuator on the total grating reflection characteristics (of both amplitude and phase).

The optical functionality of the design has been validated using this numerical model of the fabricated device for the two-grating configuration of Fig. 1. The $60 \mu \mathrm{m}$ separation between the actuators in the chip design permits the $80 \mu \mathrm{m}$ longitudinal-strain profile of each actuator to partially overlap with that of the adjacent one, thus providing a "smoothened" longitudinal refractive-index modulation profile. Figure 3 shows an example of a calculated pulse-shaper response for a $20 \mathrm{~nm}$ bandwidth and $4.5 \mathrm{~mm}$ long CFBG when a sinusoidal modulating current pattern is applied along the 75-element MEMS array: $P(N)=150[\mathrm{~mW}]$ $\sin (2 \pi N / 7)+150[\mathrm{~mW}]$, where $N$ is the actuator number 

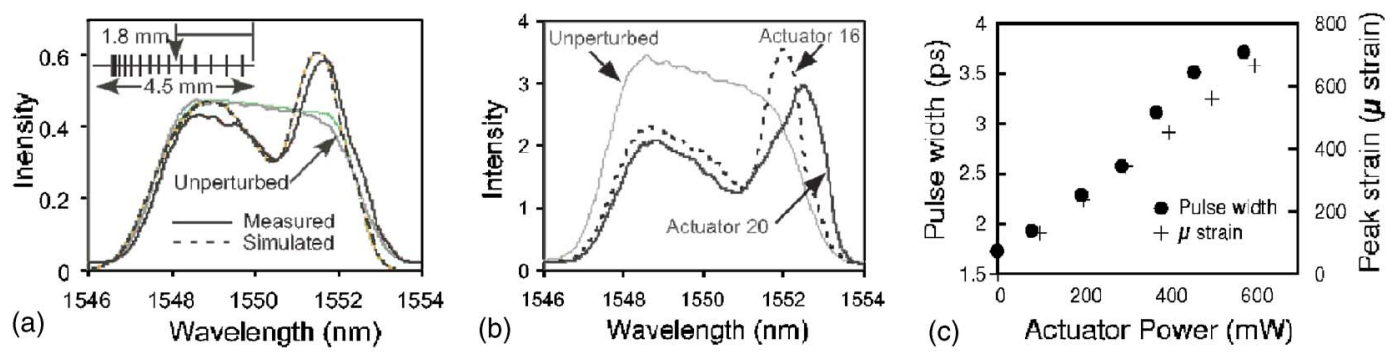

FIG. 4. (a) Comparison between measured and numerically predicted fiber-grating spectral responses to the action of a single electrothermal actuator (black traces). The actuator position is shown in the insert. Gray traces show reflection spectra of the unperturbed grating. For both perturbed and unperturbed cases, solid lines correspond to measured, while dashed lines correspond to modeled responses. (b) Measured reflection spectra, showing distinct difference in the spectral responses when actuators at different longitudinal positions are engaged. Actuators 16 and 20 are separated by $240 \mu \mathrm{m}$. (c) Autocorrelation-measured pulse duration dependence vs applied strain. The full width at half maximum (FWHM) of the optical pulses increases from 1.5 to over 4 ps, with increase in the microactuator power. The corresponding simulated peak strains in the fiber are shown.

along the array. The calculated phase response is also periodic, and the temporal shaped-pulse profile shows a generated sequence of pulses, as expected with a periodic phase modulation. This example illustrates that complex and controllable phase responses indeed can be achieved by individually controlling each of the 75 actuators.

The primary objective of the present work was to verify experimentally that the optical response of a chirped fiber grating can be accurately controlled by the micromachined actuator array. We did this by comparing responses predicted by the numerical model with experimental measurements. Indeed, for the action of a single actuator shown in Fig. 4(a), these are in very good agreement. The position of the single actuator with respect to the chirped grating is noted in the accompanying insert. By applying very large strain through the actuator, the grating reflection spectrum was significantly modified at the spectral position approximately corresponding to the actuator longitudinal position. Such comparison has been performed for various actuator positions operating at a variety of driving powers. Good agreement-similar to the one shown in Fig. 4(a)-has been observed in all these cases, proving that indeed a reproducible and accurately controlled chirped grating response has been achieved in this fiber-MEMS integrated device.

Figure 4(b) shows the optical spectrum obtained by the activation of actuators 16 and 20 along the length of the grating, illustrating the distinct changes in optical spectrum obtained using actuators only $240 \mu \mathrm{m}$ apart when activated with $500 \mathrm{~mW}$ of power. The distinct optical spectrum obtained by the use of separate actuators confirms that the spatially separated actuators are capable of addressing distinct portions of the optical spectrum.

We also verified that the thermal sensitivity of a CFBG (Ref. 10) played no role in the observed optical responses. First, modeling shows that the temperature at the probe tip of the actuator is close to room temperature, as most of the heat generated is conducted away to the substrate. Second, in the "worst-case" test a needle heated to about $350{ }^{\circ} \mathrm{C}$ was brought into contact with the CFBG, which produced no noticeable change in the optical spectrum. ${ }^{11,12}$

Changes in the temporal shape of an optical pulse caused by the action of a single actuator have been also measured using standard second-harmonic autocorrelation technique. In the experimental setup, the CFBG pair was connected in a reciprocal configuration as shown in Fig. 1. Figure 4(c) shows the observed pulse temporal broadening from
1.5 to 4 ps occurring as the pressure applied through the actuator is increased.

In summary, we have proposed and demonstrated feasibility of an integrated on-chip optical pulse shaper suitable for programmable wave form generation with ultrashort pulses. Good correspondence between numerically predicted and experimentally observed chirped fiber grating spectral responses to the action of an electrothermal actuator confirms that accurate and reproducible optical control can be achieved with this technological platform. It also indicates that the numerically predicted $\sim 1 \mu$ Strain $/ 1 \mathrm{~mW}$ fiber core strain sensitivity to the actuator driving power has been achieved in this particular implementation. More generally, the demonstrated technological platform of MEMS control of internal fiber properties can be extended to other types of devices, such as fiber couplers, long-period gratings, etc., thus enabling a new broad class of functionally diverse fiberMEMS integrated devices.

This work was partially supported by the NSF Physics Frontier Center FOCUS at the University of Michigan, Ann Arbor, and University of Texas, Austin. Facilities used for this research include the Solid-State Electronics Laboratory (SSEL) at the University of Michigan. The authors would like to thank Dr. Shamus McNamara for assistance in fabrication.

${ }^{1}$ H. Rabitz, Science 299, 525 (2003).

${ }^{2}$ N. Bonadeo, J. Erland, D. Gammon, D. Park, D. Katzer, and D. Steel, Science 282, 1473 (1998).

${ }^{3}$ A. Weiner, Rev. Sci. Instrum. 71, 1929 (2000).

${ }^{4}$ D. T. Neilson, R. Ryf, F. Pardo, V. A. Aksyuk, M.-E. Simon, D. O. Lopez, D. M. Marom, and S. Chandrasekhar, J. Lightwave Technol. 22, 101 (2004).

${ }^{5}$ R. Kashyap, Fiber Bragg Gratings (Academic, San Diego, CA, 1999).

${ }^{6}$ L. Que, J. S. Park, and Y. B. Gianchandani, J. Microelectromech. Syst. 10, 247 (2001).

${ }^{7}$ P. Gianino and B. Bendow, Appl. Opt. 20, 430 (1981).

${ }^{8}$ O. Frazão, M. J. N. Lima, and J. H. Santos, J. Opt. A, Pure Appl. Opt. 5, 183 (2003).

${ }^{9}$ K. Winick, Appl. Opt. 31, 757 (1992).

${ }^{10}$ A. D. Kersey and T. A. Berkoff, IEEE Photonics Technol. Lett. 4, 1183 (1992).

${ }^{11}$ K. Udeshi, K.-H. Liao, L. Que, A. Galvanauskas, and Y. B. Gianchandani, 18th IEEE International Conference on MEMS, 2005, pp. 239-242.

${ }^{12}$ K.-H. Liao, K. Udeshi, L. Que, Y. B. Gianchandani, and A. Galvanauskas, SPIE Photonics West, 2005 (unpublished), paper 5709-02.

${ }^{13} \mathrm{~A}$ portion of this work has been published in conference abstract form in Refs. 11 and 12. 